\title{
Origin Invariant Full Optical Rotation Tensor in the Length Dipole Gauge without London Atomic Orbitals
}

\author{
Marco Caricato ${ }^{1, a)}$ \\ Department of Chemistry, University of Kansas, Lawrence, KS 66045
}

(Dated: 7 April 2021)

We present an origin-invariant approach to compute the full optical rotation tensor (Buckingham/Dunn tensor) in the length dipole gauge without recourse to London atomic orbitals, called LG(OI). The LG(OI) approach is simpler and less computationally demanding than the more common LG-London and modified velocity gauge (MVG) approaches and it can be used with any approximate wave function or density functional method. We report an implementation at coupled cluster with single and double excitations level (CCSD), for which we present the first simulations of the origin-invariant Buckingham/Dunn tensor in the length gauge. With this method, we attempt to decouple the effects of electron correlation and basis set incompleteness on the choice of gauge for optical rotation calculations on simple test systems. The simulations show a smooth convergence of the LG(OI) and MVG results with the basis set size towards the complete basis set limit. However, these preliminary results indicate that CCSD may not be close to a complete description of the electron correlation effects on this property even for small molecules, and that basis set incompleteness may be a less important cause of discrepancy between choices of gauge than electron correlation incompleteness.

\section{INTRODUCTION}

Optically active compounds are able to rotate the plane of polarization of impinging light, a phenomenon called optical rotation (OR). ${ }^{1}$ For isotropic systems (e.g., a gas or solution phase sample), only a spatially averaged OR can be measured, often expressed as a normalized quantity known as specific rotation. However, for oriented systems such as chiral crystals, one can measure the OR in a specific direction. ${ }^{2,3}$ Simulations can in principle provide a direct comparison with experimental measurements by evaluating the Buckingham/Dunn optical activity tensor. ${ }^{4}$ Such comparison can be used to obtain structure-property relationships, assign the absolute configuration of the compound, and study the effect of intermolecular interactions on the optical activity.

Accurate quantum mechanical (QM) methods based on density functional theory (DFT) and coupled cluster (CC) theory have been developed for the calculation of chiroptical properties, ${ }^{5-20}$ using response theory to evaluate the appropriate OR tensor. ${ }^{15,19-24}$ However, given the steep computational scaling of electronic QM calculations, all of these methods only provide an approximate solution to the Schrödinger equation using an incomplete basis expansion for the electron density. Thus, the numerical results depend on the choice of gauge for the electric dipole and quadrupole operators. ${ }^{15,25-27}$ Two typical choices are the length gauge $(\mathrm{LG})$, which is more intuitive but provides an origin-dependent tensor, and the velocity gauge (VG), with which the OR tensor is origin-invariant but has an unphysical static limit. ${ }^{17,24}$ For the LG and variational methods such as HartreeFock (HF) and DFT, the origin-dependence issue is resolved using London orbitals, also known as gauge includ-

\footnotetext{
a) Electronic mail: mcaricato@ku.edu.
}

ing atomic orbitals (GIAOs). ${ }^{6,19,28,29}$ However, GIAOs cannot be utilized with standard CC methods because orbital relaxation is neglected to avoid unphysical poles in the linear response (LR) function due to the reference wave function. ${ }^{21,22}$ On the other hand, the modified VG $(\mathrm{MVG})^{17,24}$ recipe requires the explicit evaluation and removal of the unphysical static limit, and so far it has been the preferred approach for OR calculations at $\mathrm{CC}$ level. ${ }^{15,16,30,31}$

We recently proposed a strategy to overcome the origin-dependence issue of LG calculations of specific rotation without the complication of London orbitals, an approach we called LG(OI). ${ }^{32}$ This is based on a transformation of the electric dipole-magnetic dipole polarizability tensor using the singular value decomposition (SVD) eigenvectors of the mixed-gauge electric dipole-electric dipole polarizability tensor. The $\mathrm{LG}(\mathrm{OI})$ approach is simpler than the LG-GIAOs approach and faster than the MVG approach, but it shares with the latter the applicability to any approximate method. Therefore, we were able to present the first origin-invariant LG simulations of specific rotation with standard $\mathrm{CC}$ methods.

In this work, we extend the $\mathrm{LG}(\mathrm{OI})$ approach to the calculation of the full Buckingham/Dunn tensor and present the first origin-invariant LG-CC simulations of the full OR tensor. Since we now have two fully origininvariant approaches to evaluate the OR of chiral systems at $\mathrm{CC}$ level, LG(OI) and MVG, we also explore the effect of basis set and electron correlation incompleteness. We decouple these two sources of approximation using a model system where $\mathrm{CC}$ with single and double excitations (CCSD) is exact and another system where CCSD is not exact but we can use fairly large basis sets and extrapolate to the complete basis set limit. The paper is organized as follows: the theory derivation is presented in section II, details of the calculations are reported in section III, the results of numerical simulations are discussed in section IV, and concluding remarks are summarized in 
section $\mathrm{V}$.

\section{THEORY}

In this section, we present the theory for the evaluation of the full OR tensor with the LG(OI) approach. ${ }^{32} \mathrm{In}$ order to do that, it is useful to briefly review the equations for the Buckingham/Dunn optical activity tensor $\mathcal{B}$, which is defined as: ${ }^{4,23,33}$

$$
\begin{gathered}
\mathcal{B}_{\alpha \beta}=\frac{1}{2}\left[\operatorname{Tr}(\mathbf{B}) \delta_{\alpha \beta}-B_{\alpha \beta}\right] \\
B_{\alpha \beta}=\frac{1}{2}\left[\beta_{\alpha \beta}+\beta_{\beta \alpha}+\frac{1}{3} \sum_{\delta, \gamma}\left(\epsilon_{\alpha \gamma \delta} A_{\gamma, \delta \beta}+\epsilon_{\beta \gamma \delta} A_{\gamma, \delta \alpha}\right)\right]
\end{gathered}
$$

where $\boldsymbol{\epsilon}$ is the Levi-Civita operator, $\boldsymbol{\beta}$ is the electric dipole-magnetic dipole polarizability tensor, and $\mathbf{A}$ is the electric dipole-electric quadrupole polarizability tensor:

$$
\begin{aligned}
\beta_{\alpha \beta} & =2 \sum_{j \neq 0} \operatorname{Im} \frac{\left\langle\psi_{0}\left|\mu_{\alpha}\right| \psi_{j}\right\rangle\left\langle\psi_{j}\left|m_{\beta}\right| \psi_{0}\right\rangle}{\omega_{j}^{2}-\omega^{2}} \\
A_{\alpha, \beta \gamma} & =2 \sum_{j \neq 0} \omega_{j} \operatorname{Re} \frac{\left\langle\psi_{0}\left|\mu_{\alpha}\right| \psi_{j}\right\rangle\left\langle\psi_{j}\left|\Theta_{\beta \gamma}\right| \psi_{0}\right\rangle}{\omega_{j}^{2}-\omega^{2}}
\end{aligned}
$$

We use atomic units throughout the paper, except when otherwise specified. Since there has been some confusion in the literature for the use of the $\mathbf{G}^{\prime}$ symbol to indicate the electric dipole-magnetic dipole polarizability with or without the $\omega^{-1}$ factor, we use the notation $\boldsymbol{\beta}=-\omega^{-1} \mathbf{G}^{\prime}{ }^{23}$ The multipole operators in Eqs. 3-4 are expressed in the length dipole gauge as:

$$
\begin{aligned}
& \boldsymbol{\mu}=-\boldsymbol{r} \\
& \boldsymbol{m}=\frac{i}{2}(\boldsymbol{r} \times \nabla) \\
& \Theta_{\beta \gamma}=-\frac{1}{2}\left(3 r_{\beta} r_{\gamma}-\delta_{\beta \gamma} r^{2}\right)
\end{aligned}
$$

which are respectively the electric dipole, the magnetic dipole, and the traceless electric quadrupole operators, with the position $\boldsymbol{r}$ and gradient $\nabla$ operators implicitly summed over all the electrons of the molecule. The Greek indices denote Cartesian coordinates, $\omega$ is the frequency of the incident electromagnetic radiation while $\left|\psi_{j}\right\rangle$ and $\omega_{j}$ are the $j^{\text {th }}$ excited state wave function and excitation frequency, respectively. These definitions are valid for non-resonant optical activity $\left(\omega_{j} \not \omega\right)$ calculations; resonant optical activity is discussed in greater detail

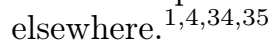

For isotropic media, the observed optical rotation is commonly reported as a normalized quantity in units of $\operatorname{deg}[\mathrm{dm}(\mathrm{g} / \mathrm{mL})]^{-1}$, known as specific rotation:

$$
[\alpha]_{\omega}=\frac{\left(72 \times 10^{6}\right) \hbar^{2} N_{A} \omega^{2}}{c^{2} m_{e}^{2} M} \operatorname{Tr}(\boldsymbol{\beta})
$$

where $\boldsymbol{\beta}$ and $\omega$ are given in atomic units, $\hbar$ is the reduced Planck's constant (J s), $N_{A}$ is Avogadro's number, $c$ is the speed of light $(\mathrm{m} / \mathrm{s}), m_{e}$ is the electron rest mass $(\mathrm{kg})$, and $M$ is the molecular mass (amu). In Eq. 6, we used the fact that the $\mathbf{A}$ contribution to $\mathbf{B}$ is traceless so that $\operatorname{Tr}(\boldsymbol{B})=\operatorname{Tr}(\mathbf{B})=\operatorname{Tr}(\boldsymbol{\beta})$. Therefore, the $\mathbf{A}$ tensor is unnecessary in OR simulations of molecules in isotropic media.

The tensors $\boldsymbol{\beta}$ and $\mathbf{A}$ in Eqs. 3-4 are both origin dependent according to:

$$
\begin{aligned}
\beta_{\alpha \beta}\left(\boldsymbol{O}^{\prime}\right)= & \beta_{\alpha \beta}(\boldsymbol{O})+\frac{1}{2} \epsilon_{\beta \gamma \delta} \alpha_{\alpha \gamma}^{(R, P)} d_{\delta} \\
A_{\alpha, \beta \gamma}\left(\boldsymbol{O}^{\prime}\right)= & A_{\alpha, \beta \gamma}(\boldsymbol{O})-\frac{3}{2} \alpha_{\alpha \gamma}^{(R, R)} d_{\beta} \\
& -\frac{3}{2} \alpha_{\alpha \beta}^{(R, R)} d_{\gamma}+\alpha_{\alpha \delta}^{(R, R)} d_{\delta} \delta_{\beta \gamma}
\end{aligned}
$$

where a sum over common indices is implied in the last term on the right-hand side of both equations, $\boldsymbol{O}$ is a particular choice of origin of the coordinate system and

$$
\boldsymbol{O}^{\prime}=\boldsymbol{O}+\boldsymbol{d}
$$

is a displaced origin, $\boldsymbol{\delta}$ is the Kronecker delta tensor, and $\alpha$ is the electric dipole-electric dipole polarizability tensor expressed with two gauge representations for the dipole operator: the superscript $(R, R)$ in Eq. 8 indicates the length gauge representation for both occurrences of the dipole operator (shown in Eq. 5), while the superscript $(R, P)$ in Eq. 7 indicates a mixed representation with the length gauge for one occurrence of the dipole operator and the velocity gauge for the other one,

$$
\boldsymbol{\mu}^{P}=-\boldsymbol{p} \text {. }
$$

where $\boldsymbol{p}$ is the momentum operator including an implicit summation over the number of electrons in the molecule. In an exact calculation, the individual origin dependent terms for the two tensors $\boldsymbol{\beta}$ and $\mathbf{A}$ perfectly cancel out when they are combined in $\mathbf{B}$ in Eq. 2 such that the latter is origin invariant. However, this is no longer the case in approximate calculations and the individual elements of $\mathbf{B}$ as well as its trace are origin dependent.

There are typically two ways to overcome the origindependence issue in approximate calculations. For the length gauge and variational methods (like HF and DFT), one can use London orbitals ${ }^{6,28}$ (also known as GIAOs), but such strategy is not feasible with conventional CC methods because the response of the molecular orbitals is neglected to avoid unphysical poles in the response function. ${ }^{21}$ An approach that works with any approximate method is to express the electric multipoles in the velocity gauge, see Eq. 10, and calculate $\boldsymbol{\beta}$ and $\mathbf{A}$ tensors accordingly. ${ }^{17,24}$ The downside of this approach is that it has an unphysical static limit that needs to be evaluated explicitly and subtracted out, a procedure known as modified velocity gauge (MVG). ${ }^{17,24}$ Note that 
the LG-GIAOs and MVG approaches provide different numerical values of the $\mathcal{B}$ tensor and specific rotation for approximate methods, and that MVG is computationally more expensive than the LG-GIAOs approach.

However, it is possible to obtain an origin-invariant version of the $\mathcal{B}$ tensor in the length gauge for any approximate method without using GIAOs. The approach is based on the same transformation we suggested for the specific rotation in Ref. 32, which we called $\mathrm{LG}(\mathrm{OI})$. First, we need a form of the $\mathbf{A}$ tensor that has a qualitatively similar origin dependence as the $\boldsymbol{\beta}$ vector in Eq. 7, i.e. dependent on the mixed-gauge electric dipole-electric dipole polarizability $\boldsymbol{\alpha}^{(R, P)}$. This is accomplished by using the following form of the quadrupole operator:

$$
\begin{aligned}
\Theta_{\beta \gamma}^{V} & =\Theta_{\beta \gamma}^{r p}+\Theta_{\beta \gamma}^{p r} \\
\Theta_{\beta \gamma}^{r p} & =-\frac{1}{2}\left(3 r_{\beta} p_{\gamma}-\delta_{\beta \gamma} \boldsymbol{r} \cdot \boldsymbol{p}\right) \\
\Theta_{\beta \gamma}^{p r} & =-\frac{1}{2}\left(3 p_{\beta} r_{\gamma}-\delta_{\beta \gamma} \boldsymbol{p} \cdot \boldsymbol{r}\right)
\end{aligned}
$$

which is also used in the MVG approach to form the $\mathbf{A}$ tensor (albeit in conjunction with the velocity electric dipole). ${ }^{24}$ With this choice of quadrupole operator:

$$
A_{\alpha, \beta \gamma}^{(R, P)}=\frac{2}{\omega} \sum_{j \neq 0} \operatorname{Im} \frac{\left\langle\psi_{0}\left|\mu_{\alpha}\right| \psi_{j}\right\rangle\left\langle\psi_{j}\left|\Theta_{\beta \gamma}^{V}\right| \psi_{0}\right\rangle}{\omega_{j}^{2}-\omega^{2}}
$$

such that the $\mathbf{A}$ tensor uses a mixed-gauge representation: length for the dipole operator and velocity for the quadrupole operator. This form of the $\mathbf{A}$ tensor preserves the expression of the $\mathbf{B}$ tensor in Eq. 2 but leads to the following expression of its origin dependence:

$$
\begin{aligned}
A_{\alpha, \beta \gamma}^{(R, P)}\left(\boldsymbol{O}^{\prime}\right)= & A_{\alpha, \beta \gamma}^{(R, P)}(\boldsymbol{O})-\frac{3}{2} \alpha_{\alpha \gamma}^{(R, P)} d_{\beta} \\
& -\frac{3}{2} \alpha_{\alpha \beta}^{(R, P)} d_{\gamma}+\alpha_{\alpha \delta}^{(R, P)} d_{\delta} \delta_{\beta \gamma}
\end{aligned}
$$

which is now compatible with that of the $\boldsymbol{\beta}$ tensor in Eq. 7. However, the use of the mixed-gauge form of the A tensor is not sufficient to lead to an origin-invariant $\mathcal{B}$ tensor because the $\boldsymbol{\alpha}^{(R, P)}$ tensor is not symmetric. Therefore, the contributions of the $\boldsymbol{\beta}$ and $\mathbf{A}^{(R, P)}$ tensors to the $\mathbf{B}$ tensor do not perfectly cancel out. To achieve that, we diagonalize $\boldsymbol{\alpha}^{(R, P)}$ tensor with a singular value decomposition (SVD):

$$
\boldsymbol{\alpha}^{(R, P)}=\boldsymbol{U} \boldsymbol{\alpha}_{D}^{(R, P)} \boldsymbol{V}^{\dagger}
$$

where $\boldsymbol{\alpha}_{D}^{(R, P)}$ is diagonal, and $\boldsymbol{U}$ and $\boldsymbol{V}^{\dagger}$ are unitary transformations. We then apply the inverse transformation to the $\boldsymbol{\beta}$ and $\mathbf{A}^{(R, P)}$ tensors:

$$
\begin{gathered}
\tilde{\beta}_{\alpha \beta}=\beta_{\alpha^{\prime} \beta^{\prime}} U_{\alpha^{\prime} \alpha} V_{\beta^{\prime} \beta} \\
\tilde{A}_{\alpha, \beta \gamma}^{(R, P)}=A_{\alpha^{\prime}, \beta^{\prime} \gamma^{\prime}}^{(R, P)} U_{\alpha^{\prime} \alpha} V_{\beta^{\prime} \beta} V_{\gamma^{\prime} \gamma}
\end{gathered}
$$

Using the $\tilde{\boldsymbol{\beta}}$ and $\tilde{\mathbf{A}}^{(R, P)}$ tensors in Eqs. 1-2 makes the $\mathcal{B}$ tensor fully origin invariant without recourse to GIAOs. The transformations in Eqs. 15-16 are sensitive to the phase of the eigenvectors and their order in the unitary matrices. Therefore, it is important to avoid inadvertently changing the handedness of the coordinate system with the transformation.

We know that the LG and VG approaches are only equivalent for an exact calculation and that approximations coming from electron correlation and basis set incompleteness in practical simulations lead to different numerical results with the two gauge choices. However, the effects of the two types of incompleteness are difficult to disentangle. Nevertheless, it would be desirable to have a criterion to compare the quality of two different calculations on the same system, at least within the same choice of gauge. For the velocity gauge, a criterion could be the relative magnitude of the unphysical static limit compared to that of the MVG result. For the LG approach, a criterion could be based on the lack of origin invariance of the specific rotation, which is related to the degree of asymmetry of the mixed-gauge polarizability tensor $\boldsymbol{\alpha}^{(R, P)}$. This degree of asymmetry can be expressed as:

$$
\Delta_{a s}=1-\frac{\left\|\boldsymbol{\alpha}_{A}^{(R, P)}\right\|_{F}}{\left\|\boldsymbol{\alpha}^{(R, P)}\right\|_{F}}
$$

where $\|\cdot\|_{F}$ represents the Frobenius norm of a matrix and the subscript $A$ indicates the anti-symmetric part of the $\boldsymbol{\alpha}^{(R, P)}$ tensor. The systematic improvement of a chosen model chemistry should correspond to an increase of $\Delta_{a s}$ towards 1 .

A legitimate question about the $\mathrm{LG}(\mathrm{OI}) \mathcal{B}$ tensor is what orientation of the molecule corresponds to this tensor values. In an exact calculation, $\boldsymbol{\alpha}^{(R, P)}$ is symmetric and $\boldsymbol{U} \equiv \boldsymbol{V}$ in Eq. 14. Therefore, the transformed $\mathcal{B}$ tensor corresponds to an orientation where the $\boldsymbol{\alpha}^{(R, P)}$ tensor is diagonal. For approximate calculations, the closest we can get to this orientation is to use the eigenvectors that diagonalize the symmetric part of the $\boldsymbol{\alpha}^{(R, P)}$ tensor, which we collect in the unitary matrix $\boldsymbol{W}$. As we approach the exact solution $\boldsymbol{U}, \boldsymbol{V} \rightarrow \boldsymbol{W}$. Once the molecule is reoriented according to the rotation defined by the $\boldsymbol{W}$ matrix, we can analytically calculate the origin displacement vector that makes the diagonal elements of the LG $\boldsymbol{\beta}$ tensor equal to the corresponding elements of the LG(OI) $\tilde{\boldsymbol{\beta}}$ tensor, so that they provide the same value of specific rotation. Using Eq. 7, the components of this particular displacement vector $\boldsymbol{d}$ are the solution of the following linear system of equations:

$$
\left\{\begin{array}{l}
\beta_{x x}^{S}\left(\boldsymbol{O}^{\prime}\right) \equiv \tilde{\beta}_{x x}^{S}=\beta_{x x}^{S}(\boldsymbol{O})+\frac{1}{2}\left(d_{z} \alpha_{x y}-d_{y} \alpha_{x z}\right) \\
\beta_{y y}^{S}\left(\boldsymbol{O}^{\prime}\right) \equiv \tilde{\beta}_{y y}^{S}=\beta_{y y}^{S}(\boldsymbol{O})+\frac{1}{2}\left(d_{x} \alpha_{y z}-d_{z} \alpha_{y x}\right) \\
\beta_{z z}^{S}\left(\boldsymbol{O}^{\prime}\right) \equiv \tilde{\beta}_{z z}^{S}=\beta_{z z}^{S}(\boldsymbol{O})+\frac{1}{2}\left(d_{y} \alpha_{z x}-d_{x} \alpha_{z y}\right)
\end{array}\right.
$$


The superscript $S$ serves as a reminder that the molecule is oriented according to the $\boldsymbol{W}$ rotation matrix and we neglected the $(R, P)$ superscript on the polarizability tensor elements for clarity. The LG $\mathcal{B}$ tensor obtained with this choice of molecular coordinates is the closest to the $\mathrm{LG}(\mathrm{OI})$ result and can be considered the best LG result obtainable for a particular approximate model chemistry.

In terms of computational cost, the $\mathrm{LG}(\mathrm{OI})$ approach is more efficient than both the LG-GIAOs and MVG approaches because no extra terms due to GIAOs are necessary and there is no static limit to evaluate explicitly. All tensors can be computed using standard linear response theory, where a perturbed density is evaluated due to length gauge electric dipole perturbation and then contracted with the appropriate multipole integrals to obtain $\boldsymbol{\beta}, \mathbf{A}^{(R, P)}$, and $\boldsymbol{\alpha}^{(R, P)} \cdot{ }^{15,19-23}$ After these tensors are available, the $\mathrm{LG}(\mathrm{OI})$ transformations in Eqs. 14-16 are trivial. For the implementation at CC level, one would need to use the non-symmetric form of the LR function, which requires the evaluation of the perturbed $T$ and $\Lambda$ amplitudes for one perturbation. ${ }^{22,36}$ In that case, the $\mathrm{LG}(\mathrm{OI})$ method is completely equivalent in cost to a standard LG calculation and less demanding than an MVG calculation. However, in our current implementation in GAUSSIAN ${ }^{37}$ at CCSD level only the symmetric form of the LR function is available, which requires the evaluation of the perturbed $T$ amplitudes for all perturbations. ${ }^{22,36}$ This route makes the $\mathrm{LG}(\mathrm{OI})$ and MVG approaches equivalent in cost. Nevertheless, this is an issue of the implementation and not of the method per se, and it will be resolved in future versions of the software.

\section{COMPUTATIONAL DETAILS}

All calculations were performed with a development version of the GAUSSIAN suite of programs. ${ }^{37}$ The tensors were computed using standard LR theory at CCSD level with frozen core orbitals for the correlation energy evaluation and frozen orbitals for the LR function. The aug-cc-pVDZ ${ }^{38,39}$ basis set was used for the three largest systems, where geometries were taken from Ref. 32 but reoriented as described in section II. For the twoelectron model system, the aug-cc-pVXZ $\mathrm{Z}^{38-40}$ and augmcc-pVYZ ${ }^{41,42}$ basis sets were used, with $X=2-6$ and $Y=7,8$ (basis sets with $X=7,8$ were not available on the basis set exchange for $\mathrm{H}) .{ }^{43-45}$ For (-)-hydrogen peroxide, a series of Dunning and Pople ${ }^{46-50}$ basis sets are used as detailed in the next section. Results are presented with the LG, LG(OI), and MVG approaches at the sodium $D$ line, i.e. $589.3 \mathrm{~nm}$, because it is far from resonance for all systems.

\section{RESULTS}

As discussed in section II, the difference between the $\mathrm{LG}$ and $\mathrm{VG}$ results is due to approximations in the level of theory used to compute the electron density (i.e., electron correlation incompleteness) and to the incompleteness of practical basis sets. These two factors are difficult to disentangle in calculations on typical molecules. Therefore, we start our discussion with a toy 2-electron model, $\mathrm{H}_{4}^{2+}$, for which CCSD provides the exact answer for a non-relativistic molecular Hamiltonian within the Born-Oppenheimer approximation (we also neglect vibrational contributions). With this model, we can directly study the effect of the basis set on the specific rotation $\left([\alpha]_{D}\right.$ is a more convenient quantity than the whole OR tensor for this analysis). We built the model by using the $\mathrm{H}_{2} \mathrm{O}_{2}$ geometry ${ }^{32}$ and replacing the Os with $\mathrm{Hs}$, and by making two somewhat random choices of origin: O1 is the origin where the LG and LG(OI) approaches provide the same result for the specific rotation of $\mathrm{H}_{2} \mathrm{O}_{2}$ with the aug-cc-pVDZ basis set and it is located somewhere inside the molecule, while $\mathrm{O} 2$ is displaced by -5 $\AA$ in every Cartesian direction from O1. Obviously, only the LG results are affected by the choice of origin and O1/O2 represent two points with no special meaning for this system. In Figure 1, we report the change in $[\alpha]_{D}$ for $\mathrm{H}_{4}^{2+}$ with increasing size of the basis set for all choices of gauge; the figure also reports the total CCSD energy and the $\Delta_{a s}$ factor defined in Eq. 17. All numerical values are reported in Tables S1-S2 of the supporting information (SI). The figure shows a smooth convergence of the specific rotation with the basis set size for all choices of gauge, except for VG. For the latter, there is an oscillatory behavior that starts from a value with the wrong sign for $\zeta=2\left(-290 \mathrm{deg} \mathrm{dm}^{-1}(\mathrm{~g} / \mathrm{mL})^{-1}\right)$; the unphysical static limit is still $>1 \mathrm{deg}$ with the $\zeta=5$ basis set. The LG(OI) and MVG values are always within $1 \mathrm{deg}$ from each other and are essentially converged with the $\zeta=5$ basis set. This indicates that basis set incompleteness is not a major cause of discrepancy between choices of gauge if the origin-dependence issue of the LG approach and the unphysical static limit issue of the VG approach are properly addressed. The LG approach converges to the $\mathrm{LG}(\mathrm{OI})$ value for the $\zeta=5$ basis set even with a significantly displaced geometry (O2). The convergence of the specific rotation follows the convergence of the energy, compare panels a) and b) of the figure, which supports that similar extrapolation formulas can be used to estimate the complete basis set (CBS) limit. ${ }^{31}$ The $\Delta_{a s}$ factor also increases until reaching a plateau with the $\zeta=5$ basis set consistently with the convergence of $[\alpha]_{D}$.

A more realistic but still small system to investigate the effect of electron correlation incompleteness on the specific rotation is (-)-hydrogen peroxide, whose geometry is taken from Ref. 32. Figure 2 reports the $[\alpha]_{D}$ values computed with the $\mathrm{LG}(\mathrm{OI})$ and MVG approaches with Dunning basis sets of increasing angular momentum functions up to $\zeta=5$ and estimates of the CBS limit; the 

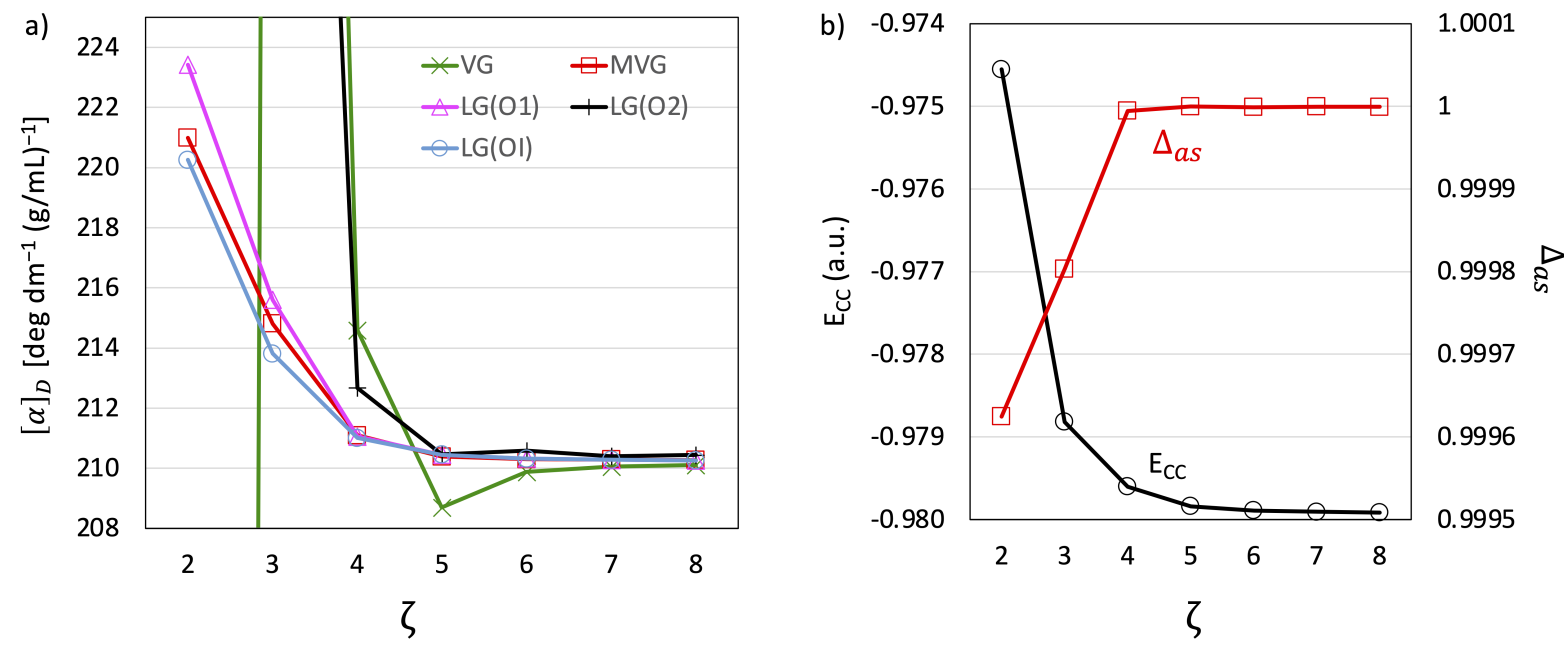

FIG. 1. Data computed for $\mathrm{H}_{4}^{2+}$ with all choices of gauge and the aug-cc-pVXZ $(X=2-6)$ and aug-mcc-pVYZ $(Y=7,8)$ basis sets at CCSD level; for LG, data are presented at two choices of the origin, O1 and O2. Panel a) specific rotation at the sodium $D$ line in $\operatorname{deg} \mathrm{dm}^{-1}(\mathrm{~g} / \mathrm{mL})^{-1}$; Panel b) combined plot of the CCSD energy ( $\mathrm{E}_{\mathrm{CC}}$ in a.u.) and $\Delta_{a s}$ factor (Eq. 17).

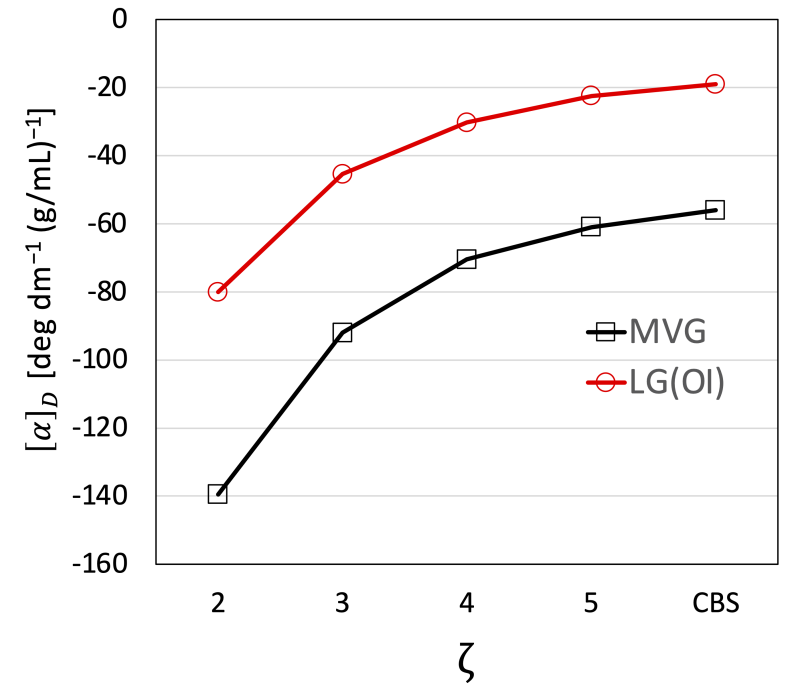

FIG. 2. Specific rotation $\left(\mathrm{deg} \mathrm{dm}^{-1}(\mathrm{~g} / \mathrm{mL})^{-1}\right)$ at the sodium D line for $\mathrm{H}_{2} \mathrm{O}_{2}$ computed with the $\mathrm{LG}(\mathrm{OI})$ and $M V G$ approaches at the CCSD/aug-cc-pV $\zeta \mathrm{Z}$ level. The CBS values are obtained with a two-point extrapolation from the $\zeta=4$ and $\zeta=5$ values (see text for details).

latter are computed with a two-point inverse-power extrapolation formula from the $\zeta=4$ and $\zeta=5$ values with exponent $n=5$ as suggested in Ref. 31. Table I reports the values in Figure 2 together with $[\alpha]_{D}$ and $\Delta_{a s}$ computed with the VG, MVG, and LG(OI) approaches for the same Dunning basis sets ${ }^{38-42}$ and a series of Pople basis sets ${ }^{46-50}$ of increasing size. From a comparison of the data in Figure 2 and Table I, the specific rotation smoothly decreases in magnitude for both the MVG and LG(OI) approaches as the size of the Dunning basis sets
TABLE I. Values of $[\alpha]_{D}\left(\operatorname{deg} \mathrm{dm}^{-1}(\mathrm{~g} / \mathrm{mL})^{-1}\right)$ and $\Delta_{a s}$ for $\mathrm{H}_{2} \mathrm{O}_{2}$ computed with the VG, MVG, and LG(OI) approaches at CCSD level and a series of Dunning and Pople basis sets.

\begin{tabular}{ccccc}
\hline \hline & & {$[\alpha]_{D}$} & & $\Delta_{a s}$ \\
& MVG & VG & LG(OI) & LG(OI) \\
\hline CBS(5Z-4Z) & -56 & -5 & -19 & - \\
aug-cc-pV5Z & -61 & -32 & -22 & 0.9989 \\
aug-cc-pVQZ & -70 & -86 & -30 & 0.9988 \\
aug-cc-pVTZ & -92 & -272 & -45 & 0.9986 \\
aug-cc-pVDZ & -140 & -899 & -80 & 0.9983 \\
6-311++G(3df,3pd) & -127 & -756 & -57 & 0.9995 \\
6-311++G(2d,2p) & -169 & -2677 & -98 & 0.9992 \\
$6-311++\mathrm{G}(\mathrm{d}, \mathrm{p})$ & -141 & -3756 & -65 & 0.9996 \\
$6-31++\mathrm{G}(\mathrm{d}, \mathrm{p})$ & -138 & -2942 & -39 & 0.9988 \\
\hline \hline
\end{tabular}

increases. Simultaneously, the VG unphysical static limit decreases in magnitude while the $\Delta_{a s}$ factor increases, as one would expect. However, the two choices of gauge do not converge to the same CBS limit, and the difference $(\sim 40 \mathrm{deg})$ is twice as large as the magnitude of the $\mathrm{LG}(\mathrm{OI})[\alpha]_{D}$ and two thirds the magnitude of the MVG $[\alpha]_{D}$. The MVG-VG difference for the CBS estimate is even larger $(\sim 50 \mathrm{deg})$. These results indicate that CCSD is not quite converged in terms of electron correlation for this molecule.

On the other hand, the Pople basis sets provide the wrong trend of $[\alpha]_{D}$ with the basis set size, as the property increases in magnitude until the largest basis set is used. This poor performance is accompanied by very large values of the static limit for the VG approach, especially for the smaller sets in the series. In contrast, the $\Delta_{a s}$ factors are as large or larger than those for the more accurate Dunning basis sets. In other words, the calculations with the Pople basis sets suffer from a smaller origin 
dependence of the $L G$ results compared to those with the Dunning basis sets even if the latter provide overall more reliable results based on the trend with basis set size.

We now report the full $\mathcal{B}$ tensor for the same three systems already used in Ref. 32 when we first introduced the LG(OI) approach: (-)-hydrogen peroxide (1), (S)-(+)2,3-pentadiene (2), and (S)-(-)-norbornenone (3). The $\mathcal{B}$ tensor values at the same geometries of Ref. 32, i.e., with the origin in the center of mass and displaced by $-1000 \AA$ in every Cartesian direction, are shown in Tables S3-S5 of the SI, to demonstrate that the LG(OI) $\tilde{\boldsymbol{\beta}}$ and $\tilde{\mathbf{A}}^{(R, P)}$ tensors combine to make a $\mathcal{B}$ tensor that is indeed origin invariant. Here, we report the data using the same geometries but rotated and translated as discussed in section II in order to maximize the agreement between the LG and LG(OI) approaches (the geometries at these orientations are reported in Tables S6-S8 of the SI). We also report the $\mathcal{B}$ tensor computed with the MVG approach for the molecules in the same orientation.

TABLE II. Values of $[\alpha]_{D}\left(\operatorname{deg} \mathrm{dm}^{-1}(\mathrm{~g} / \mathrm{mL})^{-1}\right)$ and of the $\mathcal{B}$ tensor elements (a.u.) computed with the LG, LG(OI), and MVG approaches for molecule $\mathbf{1}$ at CCSD/aug-cc-pVDZ level. The last column (\% Diff) reports the $\%$ difference between the $\mathrm{LG}(\mathrm{OI})$ and MVG results.

\begin{tabular}{ccccc}
\hline \hline & LG & LG(OI) & MVG & $\%$ Diff \\
{$[\alpha]_{D}$} & -80 & -80 & -140 & 74.3 \\
xx & -0.4202 & -0.4232 & -0.5348 & 26.4 \\
yy & 0.1096 & 0.1070 & -0.0184 & -117.2 \\
zz & 0.0993 & 0.1049 & 0.1849 & 76.2 \\
xy & 0.8200 & 0.8213 & 0.8813 & 7.3 \\
xz & 0.0000 & 0.0000 & 0.0000 & 0.0 \\
yz & 0.0000 & 0.0000 & 0.0000 & 0.0 \\
\hline \hline
\end{tabular}

TABLE III. Values of $[\alpha]_{D}\left(\operatorname{deg} \mathrm{dm}^{-1}(\mathrm{~g} / \mathrm{mL})^{-1}\right)$ and of the $\mathcal{B}$ tensor elements (a.u.) computed with the LG, LG(OI), and MVG approaches for molecule $\mathbf{2}$ at CCSD/aug-cc-pVDZ level. The last column (\% Diff) reports the $\%$ difference between the $\mathrm{LG}(\mathrm{OI})$ and MVG results.

\begin{tabular}{ccccc}
\hline \hline & LG & LG(OI) & MVG & $\%$ Diff \\
{$[\alpha]_{D}$} & 91 & 91 & 133 & 46.4 \\
xx & -2.7852 & -2.7599 & -2.6363 & -4.5 \\
yy & -0.4039 & -0.3616 & -0.1883 & -47.9 \\
zz & 3.6692 & 3.6015 & 3.5272 & -2.1 \\
xy & 5.3826 & 5.3767 & 5.1156 & -4.9 \\
xz & -0.0007 & 0.0000 & 0.0000 & 0.0 \\
yz & -0.0076 & 0.0000 & 0.0000 & 0.0 \\
\hline \hline
\end{tabular}

The data for system $\mathbf{1}$ are shown in Table II. The table compares the individual $\mathcal{B}$ tensor elements and the specific rotation for various gauges and it reports the \% difference between the LG(OI) and MVG approaches. The LG and LG(OI) values are very similar (they differ by at most $5 \%$ ), confirming that this orientation and origin location is the best obtainable for this choice of gauge at this level of theory $\left(\Delta_{a s}=0.9983\right)$. The comparison
TABLE IV. Values of $[\alpha]_{D}\left(\operatorname{deg} \mathrm{dm}^{-1}(\mathrm{~g} / \mathrm{mL})^{-1}\right)$ and of the $\mathcal{B}$ tensor elements (a.u.) computed with the LG, LG(OI), and MVG approaches for molecule $\mathbf{3}$ at CCSD/aug-cc-pVDZ level. The last column (\% Diff) reports the $\%$ difference between the $\mathrm{LG}(\mathrm{OI})$ and $\mathrm{MVG}$ results.

\begin{tabular}{ccccc}
\hline \hline & LG & LG(OI) & MVG & \% Diff \\
{$[\alpha]_{D}$} & -724 & -724 & -549 & -24.1 \\
xx & 3.1338 & 3.1392 & 2.4325 & -22.5 \\
yy & -5.6727 & -5.6737 & -4.5313 & -20.1 \\
zz & -3.5344 & -3.5388 & -2.5111 & -29.0 \\
xy & -0.0496 & -0.0491 & 0.0574 & -216.9 \\
xz & -0.8620 & -0.8546 & -0.4874 & -43.0 \\
yz & 1.0431 & 1.0483 & 0.4843 & -53.8 \\
\hline \hline
\end{tabular}

between the LG(OI) and MVG results is probably more interesting. In both cases, the largest element is xy, which is also the one for which the approaches agree best $(7 \%$ difference). The diagonal elements are different by different amounts so that the difference in specific rotation is due to cancellation effects. Although the $\mathrm{xx}$ and $\mathrm{zz}$ values are qualitatively in agreement, the yy values are not as they are computed with opposite signs. The data for system $\mathbf{2}$ is reported in Table III. For this molecule, all methods are in qualitative agreement for all tensor elements. The largest \% difference between LG(OI) and MVG is found for the smallest element in magnitude (yy with a difference of $48 \%$ ), which determines also the difference in $[\alpha]_{D}$ as the effects for the other two diagonal elements tend to cancel each other out. The asymmetry factor for this molecules is $\Delta_{a s}=0.9965$, which indicates a larger origin dependence of the LG approach compared to molecule 1. Finally, the data for system $\mathbf{3}$ are shown in Table IV. For this orientation, the specific rotation is dominated by the yy element, while the contributions from $\mathrm{xx}$ and $\mathrm{zz}$ cancel out. The relative differences in the diagonal elements between LG(OI) and MVG are pretty similar, and so it is for the specific rotation. On the other hand, the off-diagonal elements differ by about of factor of two for the $\mathrm{xz}$ and $\mathrm{yz}$ elements, although they have consistent signs. In contrast, the results for the xy element have opposite signs, but this element is one order of magnitude smaller than the others. For this system, $\Delta_{a s}=0.9994$ corresponding to a small origin dependence of the LG method (compare to the 0.9989 value of $\mathrm{H}_{2} \mathrm{O}_{2}$ with the aug-cc-pV5Z basis set in Table I).

\section{DISCUSSION AND CONCLUSIONS}

In this work, we present the theory for the origininvariant formulation of the full OR tensor using the length-dipole gauge without recourse to London atomic orbitals (also known as GIAOs). This approach, $\mathrm{LG}(\mathrm{OI})$, is based on the use of the mixed-gauge electric quadrupole operator (see Eq. 11) to evaluate the electric dipole-electric quadrupole tensor $\mathbf{A}^{(R, P)}$, and the subse- 
quent transformation of this tensor using the SVD eigenvectors of the mixed-gauge electric dipole-electric dipole polarizability tensor $\boldsymbol{\alpha}^{(R, P)}$, see Eq. 13. The LG(OI) approach is considerably less complex to implement than the common LG-GIAOs approach used in HF and DFT and it is currently the only option for origin-invariant calculations with standard LR-CC methods in the length gauge. Simultaneously, the LG(OI) approach is in principle computationally more efficient than the MVG approach because there is no unphysical static limit.

In the numerical results section (section IV), we decouple the effect of electron correlation and basis set incompleteness in the evaluation of molecular OR. Using a twoelectron model system for which CCSD is exact, we show that the $\mathrm{LG}(\mathrm{OI})$ and $\mathrm{MVG}$ approaches are equivalent even for incomplete basis sets and they smoothly converge to the CBS limit. The standard (origin-dependent) LG method also converges smoothly to the CBS limit independently of the choice of origin, but this is not the case for the VG approach where the unphysical static limit shows an erratic behavior with different basis sets, see Figure 1. The $\mathrm{H}_{2} \mathrm{O}_{2}$ example in Figure 2 shows that the LG(OI) and MVG approaches smoothly converge towards the CBS limit when using an approximate electronic structure method (CCSD). However, the two approaches converge to a significantly different CBS limit and the VG unphysical static limit is also comparatively large, see Table I, indicating that CCSD is surprisingly far from convergence in the description of electron correlation even for such a small molecule. A comparison with the results for the two-electron model system suggests that electron correlation incompleteness may be a more important cause of discrepancy between choices of gauge than basis set incompleteness. We are currently performing a thorough basis set study on multiple test molecules to explore this behavior further. Furthermore, a comparison between results obtained with Dunning and Pople basis sets in Table I indicates that while the asymmetry factor $\Delta_{a s}$ (see Eq. 17) systematically increases towards one with well-balanced basis sets (e.g., Dunning's), its value cannot be easily used as a metric for the numerical convergence of the calculations between basis sets of different type. In fact, $\Delta_{a s}$ is larger when using Pople basis sets than with Dunning basis sets even if the latter provide more reliable results.

The results for the full OR tensor $\mathcal{B}$ computed with LG(OI) and MVG in Tables II-IV indicate that the two approaches tend to provide qualitatively similar results, but the difference between the various tensor elements is not systematic. In some cases, like the yy element for molecule $\mathbf{1}$ in Table II and the xy element for molecule $\mathbf{3}$ in Table IV, the LG(OI) and MVG values have opposite signs. It is unclear at this point whether such differences are only due to the small basis set used for these calculations (aug-cc-pVDZ) or the level of theory plays a role. Also in this case, we will investigate the basis set effect in a follow-up study.

In summary, the LG(OI) approach expresses the Buck-
ingham/Dunn tensor in an origin-invariant formulation that is uniform across any level of theory, and it can be used for the evaluation of the optical rotation in oriented systems such as chiral crystals, providing a direct comparison with experimental measurements.

\section{SUPPLEMENTARY MATERIAL}

See the supplementary material for the numerical values of the quantities plotted in Figure 1 of the main text (Tables S1-S2), the values of the $\boldsymbol{\beta}, \mathbf{A}$, and $\mathcal{B}$ tensors computed with the $\mathrm{LG}(\mathrm{OI})$ approach for molecules 1-3 at the CCSD/aug-cc-pVDZ level with two choices of origin to demonstrate origin invariance (Tables S3-S5), and the Cartesian coordinates of molecules 1-3 corresponding to the tensor values in Tables II-IV of the main text (Tables S6-S8).

\section{DATA AVAILABILITY}

The data that support the findings of this study are available within the supplementary material, which contains the numerical values of the quantities plotted in Figure 1 of the main text (Tables S1-S2), the values of the $\boldsymbol{\beta}, \mathbf{A}$, and $\mathcal{B}$ tensors computed with the LG(OI) approach for molecules 1-3 at the CCSD/aug-cc-pVDZ level with two choices of origin to demonstrate origin invariance (Tables S3-S5), and the Cartesian coordinates of molecules 1-3 corresponding to the tensor values in Tables II-IV of the main text (Tables S6-S8).

\section{ACKNOWLEDGMENTS}

The author gratefully acknowledges support from the National Science Foundation through Grant No. CHE1650942 .

${ }^{1}$ L. D. Barron, Molecular Light Scattering and Optical Activity, 2nd ed. (Cambridge University Press, 2004).

${ }^{2}$ W. Kaminsky, K. Claborn, and B. Kahr, Chem. Soc. Rev. 33, 514 (2004).

${ }^{3}$ J. H. Freudenthal, E. Hollis, and B. Kahr, Chirality 21, E20 (2009).

${ }^{4}$ A. D. Buckingham and M. B. Dunn, Journal of the Chemical Society A: Inorganic, Physical, and Theoretical Chemistry , 1988 (1971).

${ }^{5}$ P. L. Polavarapu, Mol. Phys. 91, 551 (1997).

${ }^{6}$ J. R. Cheeseman, M. J. Frisch, F. J. Devlin, and P. J. Stephens, J. Phys. Chem. A 104, 1039 (2000).

${ }^{7}$ S. Grimme, Chem. Phys. Lett. 339, 380 (2001).

${ }^{8}$ P. L. Polavarapu, Chirality 14, 768 (2002).

${ }^{9}$ J. Autschbach, S. Patchkovskii, T. Ziegler, S. J. a. van Gisbergen, and E. Jan Baerends, J. Chem. Phys. 117, 581 (2002).

${ }^{10}$ K. Ruud and T. Helgaker, Chem. Phys. Lett. 352, 533 (2002).

${ }^{11}$ P. J. Stephens, D. M. McCann, J. R. Cheeseman, and M. J. Frisch, Chirality 17, 52 (2005).

${ }^{12}$ S. Grimme, A. Bahlmann, and G. Haufe, Chirality 14, 793 (2002). 
${ }^{13}$ K. Ruud, P. J. Stephens, F. J. Devlin, P. R. Taylor, J. R. Cheeseman, and M. J. Frisch, Chem. Phys. Lett. 373, 606 (2003).

${ }^{14}$ M. C. Tam, N. J. Russ, and T. D. Crawford, J. Chem. Phys. 121, 3550 (2004).

${ }^{15}$ T. D. Crawford, Theoretical Chemistry Accounts 115, 227 (2006).

${ }^{16}$ T. D. Crawford and P. J. Stephens, J. Phys. Chem. A. 112, 1339 (2008).

${ }^{17}$ T. B. Pedersen, H. Koch, L. Boman, and A. M. Sánchez de Merás, Chem. Phys. Lett. 393, 319 (2004).

${ }^{18}$ T. D. Crawford, L. S. Owens, M. C. Tam, P. R. Schreiner, and H. Koch, J. Am. Chem. Soc. 127, 1368 (2005).

${ }^{19}$ M. Krykunov and J. Autschbach, J. Chem. Phys. 123, 114103 (2005).

${ }^{20}$ J. Autschbach, Chirality 21, E116 (2009).

${ }^{21}$ H. Koch and P. Jørgensen, J. Chem. Phys. 93, 3333 (1990).

${ }^{22}$ O. Christiansen, P. Jorgensen, and C. Hattig, Int. J. Quantum Chem. 68, 1 (1998).

${ }^{23}$ J. Autschbach, ChemPhysChem 12, 3224 (2011).

${ }^{24}$ K. Zhang, T. Balduf, and M. Caricato, Chirality , Accepted, DOI:10.1002/chir.23310 (2021).

${ }^{25}$ S. Pelloni and P. Lazzeretti, J. Chem. Phys. 140, 074105 (2014).

${ }^{26}$ P. Lazzeretti, Int. J. Quantum Chem. 114, 1364 (2014).

${ }^{27}$ M. C. Caputo, S. Pelloni, and P. Lazzeretti, Int. J. Quantum Chem. 115, 900 (2015).

${ }^{28}$ F. London, J. Phys. Radium 8, 397 (1937).

${ }^{29}$ R. Ditchfield, Mol. Phys. 27, 789 (1974).

${ }^{30}$ T. J. Mach and T. D. Crawford, J. Phys. Chem. A 115, 10045 (2011).

${ }^{31}$ S. Haghdani, P.-O. Åstrand, and H. Koch, J. Chem. Theory Comput. 12, 535 (2016).

${ }^{32}$ M. Caricato, J. Chem. Phys. 153, 151101 (2020).

${ }^{33}$ T. Balduf and M. Caricato, J. Phys. Chem. C 123, 4329 (2019).

${ }^{34}$ P. Norman, K. Ruud, and T. Helgaker, J. Chem. Phys. 120, 5027 (2004).

${ }^{35}$ M. Krykunov and J. Autschbach, J. Chem. Phys. 125 (2006), $10.1063 / 1.2210474$.

${ }^{36}$ J. F. Stanton and J. Gauss, Int. Rev. Phys. Chem. 19, 61 (2000).

${ }^{37}$ M. J. Frisch, G. W. Trucks, H. B. Schlegel, G. E. Scuseria, M. A. Robb, J. R. Cheeseman, G. Scalmani, V. Barone, G. A. Petersson, H. Nakatsuji, X. Li, M. Caricato, A. V. Marenich, J. Bloino,
B. G. Janesko, R. Gomperts, B. Mennucci, H. P. Hratchian, J. V. Ortiz, A. F. Izmaylov, J. L. Sonnenberg, D. WilliamsYoung, F. Ding, F. Lipparini, F. Egidi, J. Goings, B. Peng, A. Petrone, T. Henderson, D. Ranasinghe, V. G. Zakrzewski, J. Gao, N. Rega, G. Zheng, W. Liang, M. Hada, M. Ehara, K. Toyota, R. Fukuda, J. Hasegawa, M. Ishida, T. Nakajima, Y. Honda, O. Kitao, H. Nakai, T. Vreven, K. Throssell, J. A. Montgomery, Jr, J. E. Peralta, F. Ogliaro, M. J. Bearpark, J. J. Heyd, E. N. Brothers, K. N. Kudin, V. N. Staroverov, T. A. Keith, R. Kobayashi, J. Normand, K. Raghavachari, A. P. Rendell, J. C. Burant, S. S. Iyengar, J. Tomasi, M. Cossi, J. M. Millam, M. Klene, C. Adamo, R. Cammi, J. W. Ochterski, R. L. Martin, K. Morokuma, O. Farkas, J. B. Foresman, and D. J. Fox, "Gaussian development version revision j.11," (2020).

${ }^{38}$ T. H. Dunning Jr, J. Chem. Phys. 90, 1007 (1989).

${ }^{39}$ R. A. Kendall, T. H. Dunning, and R. J. Harrison, J. Chem. Phys. 96, 6796 (1992)

${ }^{40}$ K. A. Peterson, D. E. Woon, and T. H. Dunning, Jr, J. Chem. Phys. 100, 7410 (1994).

${ }^{41}$ S. L. Mielke, B. C. Garrett, and K. A. Peterson, J. Chem. Phys. 116, 4142 (2002)

${ }^{42}$ S. L. Mielke, D. W. Schwenke, and K. A. Peterson, J. Chem. Phys. 122, 224313 (2005).

${ }^{43}$ B. P. Pritchard, D. Altarawy, B. Didier, T. D. Gibsom, and T. L. Windus, J. Chem. Inf. Model. 59, 4814 (2019).

${ }^{44}$ D. Feller, J. Comput. Chem. 17, 1571 (1996).

${ }^{45}$ K. L. Schuchardt, B. T. Didier, T. Elsethagen, L. Sun, V. Gurumoorthi, J. Chase, J. Li, and T. L. Windus, J. Chem. Inf. Model. 47, 1045 (2007)

${ }^{46}$ R. Ditchfield, W. J. Hehre, and J. A. Pople, J. Chem. Phys. 54, 724 (1971).

${ }^{47}$ P. C. Hariharan and J. A. Pople, Theor. Chim. Acta 28, 213 (1973).

${ }^{48}$ M. J. Frisch, J. A. Pople, and J. S. Binkley, J. Chem. Phys. 80, 3265 (1984).

${ }^{49}$ R. Krishnan, J. S. Binkley, R. Seeger, and J. A. Pople, J. Chem. Phys. 72, 650 (1980).

${ }^{50}$ T. Clark, J. Chandrasekhar, G. W. Spitznagel, and P. V. R. Schleyer, J. Comput. Chem. 4, 294 (1983). 